\title{
Práticas digitais para minimizar o impacto do COVID-19 nas instituições de ensino
}

\author{
Digital practices to minimize the impact of COVID-19 on educational institutions \\ Prácticas digitales para minimizar el impacto del COVID-19 en las instituciones educativas
}

Recebido: 28/11/2021 | Revisado: 01/12/2021 | Aceito: 10/12/2021 | Publicado: 18/12/2021

\author{
Arnold de Araujo Freitas \\ ORCID: https://orcid.org/0000-0003-3202-2418 \\ Universidade Federal Fluminense, Brasil \\ E-mail: arnoldfreitas@id.uff.br \\ Matheus Carvalho do Nascimento \\ ORCID: https://orcid.org/0000-0002-0041-142X \\ Universidade Federal Fluminense, Brasil \\ E-mail: matheuscn@id.uff.br \\ Geórgia Regina Rodrigues Gomes \\ ORCID: https://orcid.org/0000-0003-0907-9838 \\ Universidade Federal Fluminense, Brasil \\ E-mail: georgiagomes@id.uff.br \\ João Paulo de Oliveira Faria \\ ORCID: https://orcid.org/0000-0002-7008-8568 \\ Universidade Federal Fluminense, Brasil \\ E-mail: jpfaria25@hotmail.com
}

\begin{abstract}
Resumo
No contexto da Pandemia do Coronavírus 2020-21 os serviços mediados pelas Tecnologias da Informação e Comunicação se tornaram essenciais para diversos setores sociais, especialmente para a Educação. A viabilidade de realização de aulas online e do trabalho remoto permitiram a manutenção de atividades sem a necessidade de deslocamentos ou aglomerações. Nesse sentido, a presente pesquisa teve por objetivo analisar a percepção dos servidores técnico administrativos do Instituto do Noroeste Fluminense de Educação Superior (INFES/UFF) no que se refere à realização de atividades de origem da Tecnologia da Informação e Comunicação (TIC) e seus cotidianos durante o trabalho remoto. Esta pesquisa é caracterizada como quantitativa e descritiva, tendo utilizado como instrumento de coleta de dados um questionário, disponibilizado via grupo de Whatsapp. Os resultados obtidos demonstraram que o setor de TI da referida instituição é de suma importância para o bom desenvolvimento do trabalho dos técnicos administrativos, presencial ou remotamente. Ainda, o planejamento estratégico desse setor permitiu o bom funcionamento da estrutura do mesmo, bem como a implantação de ferramentas para viabilizar o trabalho remoto durante a pandemia, garantindo suporte eficiente aos usuários.
\end{abstract}

Palavras-chave: Pandemia; Tecnologia da informação; Governança; Gestão pública; Ensino superior; TIC.

\begin{abstract}
In the context of the Coronavirus 2020-21 Pandemic, services mediated by Information and Communication Technologies are essential for various social sectors, especially for Education. The feasibility of conducting online classes and working remotely allow the maintenance of activities without the need for displacements or crowding. In this sense, this research aimed to analyze the perception of administrative technical servants of the Instituto do Noroeste Fluminense for Higher Education (INFES / UFF) with regard to the carrying out of activities originating in Information and Communication Technology and their daily lives during the remote work. This research is characterized as quantitative and descriptive, having used a questionnaire as a data collection instrument, made available via the WhatsApp group. The results obtained showed that the institution's IT sector is of paramount importance for the good development of the work of administrative technicians, in person or remotely. Still, the strategic planning of this user's sector, the proper functioning of its structure, as well as the implementation of tools to enable remote work during a pandemic, ensuring efficient support to users.
\end{abstract}

Keywords: Pandemic; Information technology; Governance; Public management; Higher education; ICT.

\section{Resumen}

En el contexto de la Pandemia del Coronavirus 2020-21, los servicios mediados por las Tecnologías de la Información y la Comunicación son fundamentales para diversos sectores sociales, especialmente para la Educación. La viabilidad de realizar clases online y trabajar de forma remota permite el mantenimiento de actividades sin necesidad de desplazamientos ni hacinamiento. En este sentido, esta investigación tuvo como objetivo analizar la percepción de los servidores técnicos administrativos del Instituto Noroeste Fluminense de Educación Superior (INFES / UFF) con respecto a la realización de actividades originadas en las Tecnologías de la Información y la Comunicación y su vida 
cotidiana durante el remoto trabaja. Esta investigación se caracteriza por ser cuantitativa y descriptiva, habiendo utilizado un cuestionario como instrumento de recolección de datos, puesto a disposición a través del grupo de WhatsApp. Los resultados obtenidos mostraron que el sector de TI de la institución es de suma importancia para el buen desarrollo del trabajo de los técnicos administrativos, de manera presencial o remota. Aún así, la planificación estratégica del sector de este usuario, el buen funcionamiento de su estructura, así como la implementación de herramientas que permitan el trabajo remoto durante una pandemia, asegurando un apoyo eficiente a los usuarios.

Palabras clave: Tecnología de la información; Gobernanza; Gestión pública; Educación superior; TIC.

\section{Introdução}

No final de dezembro de 2019, as autoridades de saúde chinesas informaram à Organização Mundial da Saúde (OMS) sobre um grupo de pacientes com uma misteriosa pneumonia. Em 30 de janeiro de 2020, a OMS declarou o surto de COVID19, uma emergência de saúde pública de interesse internacional. Pandemia - doença contagiosa com disseminação significativa e contínua de pessoa para pessoa em vários países em todo o mundo ao mesmo tempo - de repente se tornou uma palavra familiar, inundando os noticiários e cotidianos. Nesse contexto, uma das principais estratégias adotadas para se conter a pandemia de COVID-19 foi o isolamento social, implicando em medidas que atingiram os setores sociais, econômicos, culturais, dentre outros. Diante desse cenário, as Tecnologias de Informação e Comunicação (TICs) contribuíram significativamente nesse difícil momento, permitindo o rápido acesso à informação e possibilitando a manutenção de diversos serviços, sem a necessidade de deslocamentos ou aglomerações.

Poucos dias após o início do surto, o trabalho em casa (home office) - até então praticado esporadicamente por empresas e organizações - tornou-se comum, numa questão de sobrevivência física e financeira. Para prevenir a propagação do vírus e proteger os trabalhadores, governos de todo o mundo instruíram os empregadores a fecharem seus escritórios, permitindo que seus funcionários trabalhassem à distância. Foi estimado que mais de quatro em cada cinco pessoas (81 por cento) da força de trabalho global de 3,3 bilhões foram afetadas pelo fechamento total ou parcial do local de trabalho (ILO, 2020). Grande parte dos empregadores e funcionários possuíam experiência limitada com o trabalho remoto; de repente, com a suspensão das atividades presenciais, empresas e demais organizações foram confrontadas com a real possibilidade de perder receitas e/ou gerar empregos. Combinado com o medo e a incerteza provocados pelo vírus, a interrupção das atividades presenciais foi também estressante e dolorosa (Vieira, 2020). Nesse contexto, o trabalho remoto foi visto como alternativa para a manutenção das rotinas, e uma solução potencial para aliviar a dor.

Nas últimas décadas, as TICs possibilitaram avanços e desenvolvimentos para as mais diversas áreas. As aplicações das mesmas permitiram novas estratégias de negócios para as organizações, além de cumprirem importante papel social no que diz respeito à disseminação da informação e comunicação. A pandemia de COVID-19 reforçou o espaço das tecnologias digitais no cotidiano da sociedade contemporânea. Considerando, especialmente, o setor educacional e o impacto da suspensão das atividades presencias para esse, o uso das TIC demonstrou-se essencial para que as atividades de ensino e rotinas administrativas pudessem ser mantidas. Seguindo a Instrução Normativa No 27, de 25 de março de 2020 (IN no 27/2020), as IFES (Instituições Federais de Ensino Superior) adotaram o regime de trabalho remoto na maior parte de seus setores. Com isso, houve um aumento exponencial na demanda de recursos tecnológicos para oferecer, de forma remota, as condições de execução dos procedimentos administrativos habituais. Assim, as mais diversas instituições de ensino buscaram, através de seus gestores de TI (Tecnologia da Informação), soluções inteligentes para promover a disponibilidade de serviços e informações necessárias para a continuidade de suas atividades. A Universidade Federal Fluminense (UFF), por exemplo, definiu, através da Resolução $\mathrm{n}^{\circ} 156 / 2020$ do Conselho de Ensino, Pesquisa e Extensão (CEPEx), de 12 de junho de 2020, os critérios para planejamento e execução das atividades acadêmicas emergenciais, mediadas por tecnologias digitais.

Todo esse novo cenário demandou uma nova postura por parte de educadores e demais profissionais ligados às instituições de ensino. Camila et. al. (2021) pontuam: 
"Toda a equipe envolvida com o processo de ensino aprendizagem por meio do sistema on-line da escola se deparam diante de uma realidade jamais presenciada por escolas públicas, onde o ensino era fornecido somente através das transmissões de saberes de professores e alunos dentro de uma sala de aula de ensino regular, e depois da realidade enfrentada diante o período pandêmico, tiveram que se adaptar a nova realidade por meio de instalações tecnológicas e internet de boa qualidade dentro do ambiente educacional e meios para que todos os envolvidos no processo soubessem utilizar as tecnologias para suprir a necessidade de aprendizagem do estudante (Camila et al, 2021).”

No que se refere à Gestão de TI, exigiu-se dessa maior dinamismo e flexibilidade, a fim de acompanhar as mudanças externas provocadas pela pandemia, com o objetivo de oportunizar, a seus usuários, o bom desenvolvimento de suas atividades (Laurindo, 2001).

Neste sentido, o presente trabalho teve por objetivo geral analisar a percepção dos servidores técnico administrativos de uma das IFES no Noroeste do Estado do Rio de Janeiro em relação às atividades de origem da Tecnologia da Informação e seus cotidianos durante o trabalho remoto. Para atingir tal objetivo o trabalho contou com os seguintes objetivo específicos:

- Investigar como os servidores técnico administrativos avaliavam o setor de TI e suas contribuições nas atividades presenciais (antes da pandemia);

- Analisar a percepção dos servidores técnico administrativos em relação à atuação do setor de TI para com suas atribuições profissionais durante o trabalho remoto;

- Verificar se, e como, o setor de TI contribuiu para possibilitar a atuação profissional diante da nova rotina de trabalho remoto dos servidores técnico administrativos.

\section{Referencial Teórico}

Segundo Craig et al. (2007), a TI exerce papéis distintos nos processos das instituições, dependendo do que ela é projetada a fazer. Os autores abordam a TI baseada em três papéis, com base na relação e suporte a produtos e processos do negócio, a saber: fornecedora de serviços; instrumento gerencial alinhado aos interesses do negócio; e ferramenta de inovação, ou diferenciador competitivo. No que tange ao uso dessas tecnologias nas Instituições de Ensino Superior (IES), Magalhães (2017) destaca a importância do uso da Internet para fins de pesquisa, capacitação, a infraestrutura tecnológica disponibilizada pela instituição, a disponibilidade de investimento e o grau de satisfação dos usuários com relação aos sistemas de informação, os benefícios e o alinhamento da TI com o Planejamento Estratégico da Instituição (PETI). Em razão da pandemia de COVID19, as diversas atividade educacionais presenciais foram suspensas, sendo reorganizadas e ofertadas de maneira remota, através do Ensino Remoto Emergencial (ERE), caracterizado pela adaptação das aulas presenciais com utilização das TICs para estabelecer uma comunicação síncrona com os alunos (Hodges, 2020).

A atuação remota dos profissionais da educação tornou mais evidente e importante o papel das tecnologias digitais para dar seguimento às aulas durante a pandemia. Para os servidores técnico administrativos em educação, acostumados a trabalharem presencialmente nas instituições de ensino, com toda uma infraestrutura bem definida e implementada (que possibilita o acesso à sistemas, comunicação ágil, reuniões, acessos à documentos, etc.), foi necessária a intervenção das equipes de TI para adaptar, inovar e disponibilizar, a partir das tecnologias digitais, uma nova realidade compatível com o cenário atual. Dentre as principais adaptações adotadas pelas IFES destacam-se duas: a videoconferência, "utilizado atualmente, por empresas e alguns técnicos da área, na mesma acepção do termo webconferência, pois está cada vez menos usual a videoconferência nos moldes da década de 70” (Souza \& Pimenta, 2014, p. 372), e as redes privadas virtuais (do inglês Virtual Private Networks) ou VPN.

De acordo com Baltzan (2012), a webconferência permite a utilização de ligações ou mensagens de texto em tempo real. Pode-se compartilhar documentos, vídeos, assistir a demonstrações de softwares, dentre outras possibilidades. Já as Redes Privadas Virtuais (VPNs) consistem em redes criadas sobre a rede de comunicação já existente. As VPNs possuem caráter privado pois toda a informação é transmitida de forma segura usando encriptação ou recurso a encapsulamento do tráfego por 
outro protocolo, criando desta forma um túnel entre os dois extremos da comunicação (SILVA et al., 2012). O acesso remoto é realizado por usuários móveis que se conectam à rede corporativa através de um computador, partindo de suas residências, ou a partir de qualquer lugar através de redes wireless (Rossi \& Franzin, 2000). "Uma VPN de acesso remoto pode ser usada para conectar empresas e colaboradores que estejam distantes fisicamente, e neste caso, torna-se necessário um software cliente de acesso remoto" (Silva, 2016 apud De Araújo Cardoso, 2018). Especialmente para o setor educacional, as VPNs possibilitaram o estabelecimento de uma conexão entre os profissionais da educação e suas atividades laborais, possibilitando uma atuação remota acessível e produtiva.

\section{Metodologia}

A pesquisa foi pautada em uma metodologia quantitativa, a partir da utilização de um questionário composto por 3 questões fechadas e 1 questão aberta, conforme Tabela 1. Serapioni (2000, p.188) indica que para a metodologia quantitativa “os resultados adquiridos são generalizáveis para o conjunto da comunidade", proporcionando uma análise ampla dos dados obtidos na pesquisa.

Tabela 1 - Questionário;

\begin{tabular}{|l|l|}
\hline \multicolumn{1}{|c|}{ Pergunta } & \multicolumn{1}{c|}{ Alternativa } \\
\hline $\begin{array}{l}\text { Como você avalia o setor de TI e suas contribuições nas } \\
\text { suas atividades presenciais (antes da pandemia)? }\end{array}$ & $\begin{array}{l}\text { Escala ordinal de 1 a 5 } \\
\text { Iniciando em "Muito Insatisfeito" até "Muito Satisfeito" }\end{array}$ \\
\hline $\begin{array}{l}\text { Como você avalia o setor de TI e suas contribuições nas } \\
\text { suas atividades remotas? }\end{array}$ & $\begin{array}{l}\text { Escala ordinal de 1 a 5 } \\
\text { Iniciando em "Muito Insatisfeito" até "Muito Satisfeito" }\end{array}$ \\
\hline $\begin{array}{l}\text { O setor de TI ofereceu soluções para aproximar e e } \\
\text { possibilitar a sua atuação com a nova rotina de trabalho } \\
\text { remoto. }\end{array}$ & $\begin{array}{l}\text { Escala ordinal de 1 a 5 } \\
\text { Iniciando em "Discordo totalmente" até "Concordo } \\
\text { totalmente" }\end{array}$ \\
\hline $\begin{array}{l}\text { Opine sobre o setor de TI do seu Instituto no que tange } \\
\text { ao trabalho remoto. }\end{array}$ & *Pergunta aberta \\
\hline
\end{tabular}

Fonte: Autores.

A amostra foi constituída por 23 servidores técnico administrativos do INFES (Instituto do Noroeste Fluminense de Educação Superior), unidade da UFF localizada na cidade de Santo Antônio de Pádua, RJ. O Google Forms (2021) foi utilizado como ferramenta para a elaboração do questionário e o link de aceso foi encaminhado aos técnicos administrativos através de um grupo de Whatsapp já existente, onde todos estão inseridos. Quatorze servidores retornaram o questionário, os quais foram analisados e discutidos na presente pesquisa.

\section{Resultados, Análise e Discussão}

Para as três perguntas objetivas do questionário o resultado obtido foi unânime, onde todos os entrevistados afirmaram estar muito satisfeitos em cada um dos casos, conforme mostram as Figuras 1, Figura 2 e Figura 3: 
Figura 1 - Pergunta 1.

Como vocè avalia o setor de Tl e suas contribuiçōes nas suas atividades presenciais (antes da pandemia)?

14 respostas

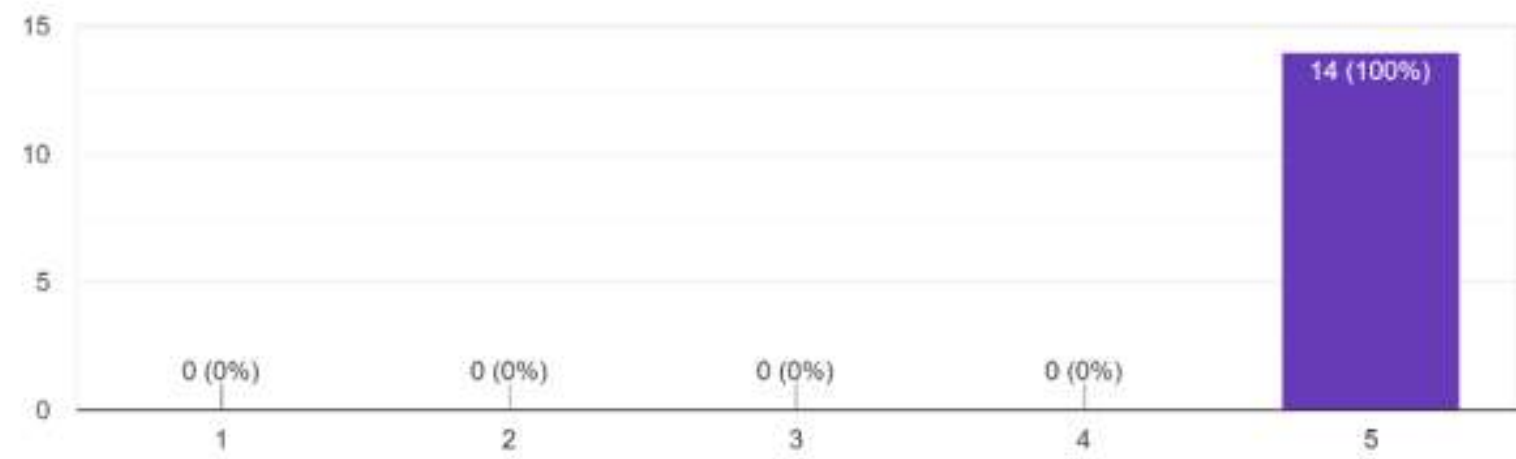

Fonte: Autores.

Figura 2 - Pergunta 2.

Como você avalia o setor de Tl e suas contribuiçōes nas suas atividades remotas?

14 respostas.

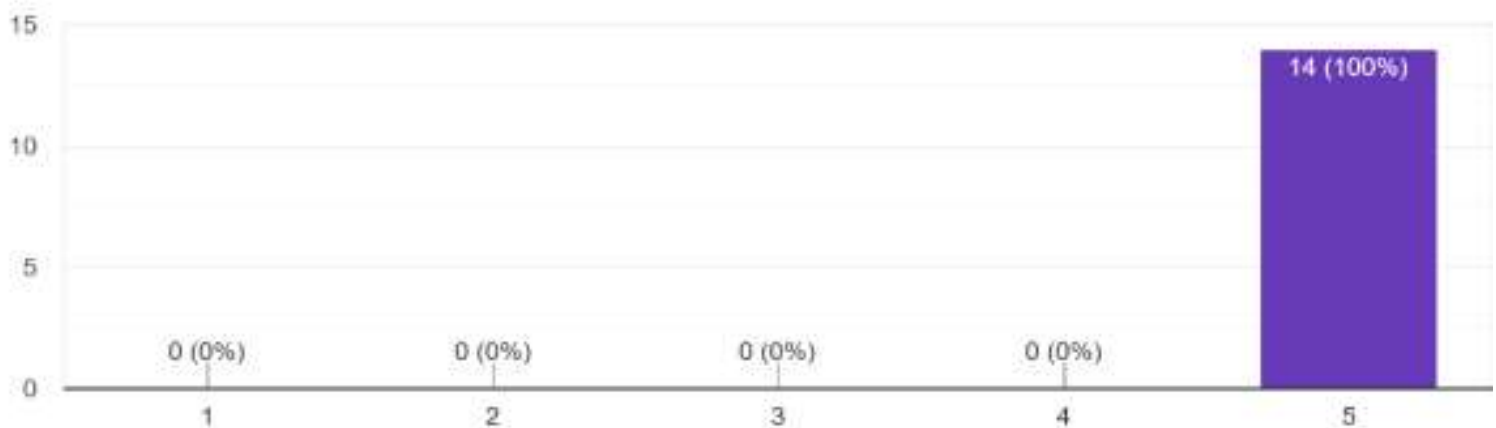

Fonte: Autores. 
Figura 3 - Pergunta 3.

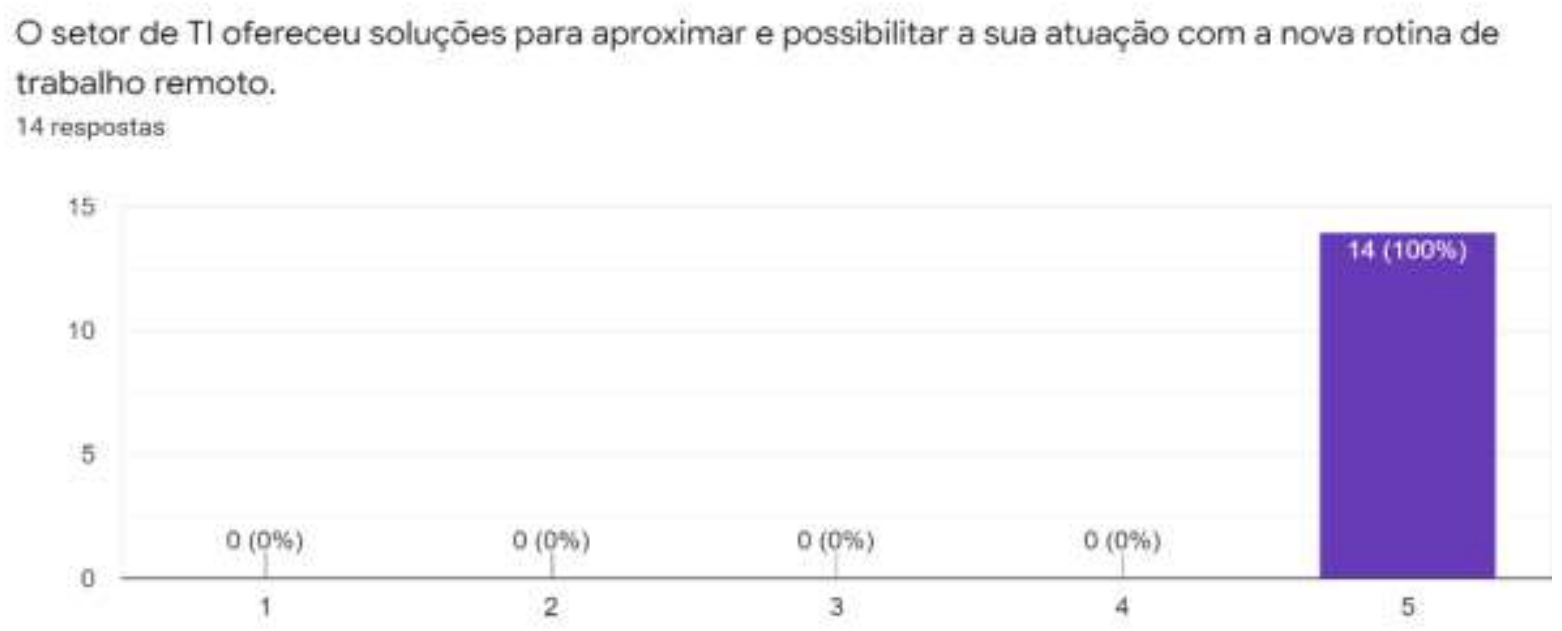

Fonte: Autores.

Os resultados obtidos nestas respostas nos mostram que o setor de TI do INFES já vinha prestando um suporte de qualidade em um período pré pandêmico, conseguindo implementar soluções que minimizassem os impactos do desenvolvimento das atividades em período remoto. Rosa, Arana \& Pereira (2021) encontraram resultados semelhantes, ressaltando o papel das tecnologias da informação de modo a acompanhar as mudanças impostas pela pandemia.

Para a pergunta aberta o resultado também foi $100 \%$ positivo, conforme alguns relatos abaixo:

"O setor de TI do INFES, mesmo antes da pandemia, já apresentava um excelente trabalho junto à comunidade acadêmica do Instituto. Durante esse período de trabalho remoto, o setor de TI continua mantendo o alto padrão de qualidade no atendimento às diversas demandas e criou um acesso, via VPN, aos arquivos dos computadores dos funcionários para que estes tenham acesso aos seus respectivos documentos, facilitando, assim, o desenvolvimento do trabalho."

"O setor de TI contribui, constantemente, com ferramentas e soluções que possibilitam a execução elou aperfeiçoam as atividades dos servidores nos diversos setores da universidade. As ações rápidas do setor no início das atividades remotas foram fundamentais para a continuidade e desenvolvimento dos trabalhos."

“A equipe de T.I. do Infes é comprometida, pró ativa e capacitada. Nunca esperei muito para ser atendida e, na verdade, o atendimento é quase sempre imediato e sempre eficaz. Muitas vezes somos avisados sobre problemas possíveis, alertados sobre perigos de mensagens e informados com antecedência sobre manutenções que nos deixarão algum tempo sem acesso aos sistemas e redes que utilizamos. Os técnicos são muito educados e atenciosos e estão sempre disponíveis. Eles respeitam e nos ajudam com nossas dificuldades, por mais simples que elas sejam. A equipe de T.I. do Infes é nota 10!"”

As manifestações dos respondentes refletem o pensamento de Adriana (2001), que nos lembra que os softwares e recursos digitais, se não associados a um bom planejamento e organização, se tornam apenas uma fonte de gasto de recursos. Ao contrário, quando estes são tratados por gestores competentes, com processos maduros e visão estratégica, as ferramentas digitais podem potencializar a produtividade da instituição. 
Deste modo, podemos dizer que o setor de TI na instituição é de suma importância para o trabalho dos técnicos administrativos, seja presencialmente ou remotamente, como ocorre durante o período de pandemia. Além das contribuições rotineiras para garantir o bom funcionamento da estrutura de TI, vimos que são de grande valia as soluções pontuais diante do cenário em que estamos vivendo. Especificamente para o caso do INFES/UFF, a implantação do acesso via VPN foi uma ferramenta crucial para o sucesso do trabalho remoto.

\section{Conclusão}

A Universidade Federal Fluminense (UFF) se insere em Santo Antônio de Pádua no ano de 1985, com a oferta inicial de apenas um curso (Licenciatura em Matemática). A UFF ofereceu atenção especial à interiorização e foi criado em 2008 o Instituto do Noroeste Fluminense de Educação Superior (INFES). Foram criados mais 7 cursos e desde 2012, o INFES expandiu consideravelmente seus quadros de docentes e técnico administrativos. As atividades de ensino, pesquisa e extensão desenvolvidas na instituição têm transformado a realidade do município de Santo Antônio de Pádua e região (FLUMINENSE, 2021).

Para acompanhar a alta demanda tecnológica e otimizar o trabalho, alinhando os serviços de TI às estratégias da instituição, foi criada a Gerência de Tecnologia da Informação (GTI). Os excelentes resultados obtidos neste estudo de caso são resultados de uma instituição onde o planejamento estratégico de TI vinha sendo realizado pela GTI antes do período de pandemia e vem se mantendo também neste período, refletindo nos resultados encontrados na presente pesquisa e no cotidiano dos servidores técnico administrativos dessa IFES. A atuação do setor corrobora com as concepções de Smitch e McKenn (2006) que, há mais de uma década, projetavam "uma tendência nos próximos anos, de a TI aumentar sua flexibilidade pela maior adaptação de suas atividades, processos e estrutura, aos interesses dos negócios".

Por fim, este trabalho permite continuidade futura, mapeando e identificando as futuras demandas dos servidores técnicos administrativos da educação no período pós pandemia, identificando as maneiras com que a atuação remota pode se refletir no retorno ao trabalho presencial.

\section{Referências}

Baltzan, P., \& Phillips, A. (2012). Sistemas de Informação. In Google Books. McGraw Hill Brasil. https://books.google.com.br/books?hl=ptBR\&lr=\&id=NJkR83DSkPYC\&oi=fnd $\&$ pg=PR5\&dq=BALTZAN

Beal, A. (2001). Introdução à gestão de tecnologia da informação. 2Beal Educação e Tecnologia. Maio de.

Brasil. MEC (2020) Universidade Federal Fluminense Conselho de Ensino, Pesquisa e Extensão.

Brasil. Ministério da Economia (2020). Instrução Normativa nº 27 de 2020. Diário Oficial [da] República Federativa do Brasil, Brasília, DF, 25 março 2020.

Brasil. Ministério da Economia Secretaria Especial de Desburocratização, Gestão e Governo Digital/Secretaria de Gestão e Desempenho de Pessoal, INSTRUÇÃO NORMATIVA No 27, DE 25 DE MARÇO DE 2020

Craig, D., Kanakamedala, K., \& Tinaikar, R. (2007). The next frontier in IT strategy: A McKinsey Survey. McKinsey Quarterly, 1-3.

De Araújo Cardoso, G., et al. (2021) Redes Privadas Virtuais: O Uso do Radmin VPN Como Solução VPN para Pequenas Empresas.

Universidade Federal Fluminense. (2021) O INFES: histórico. Histórico. http://infes.uff.br/historico/.

Hodges, C., Moore, S., Lockee, B., \& Bond, A. (2020). As diferenças entre o aprendizado online e o ensino remoto de emergência. Revista da escola, professor, educação e tecnologia, 2.

ILO: COVID-19 causes devastating losses in working hours and employment. International Labour Organization, 2020. <https://bit.ly/2XjoxFu>.

Laurindo, F. J. B., Shimizu, T., Carvalho, M. M. D., \& Rabechini Jr, R. (2001). O papel da tecnologia da informação (TI) na estratégia das organizações. Gestão \& Produção, 8, 160-179.

Magalhães, C., \& Mac-allister, M. (2017). Gestão da Tecnologia da Informação (TI) nas Instituições de Ensino Superior (IES): um estudo de caso numa IES particular de Salvador. 
Research, Society and Development, v. 10, n. 16, e538101624002, 2021

(CC BY 4.0) | ISSN 2525-3409 | DOI: http://dx.doi.org/10.33448/rsd-v10i16.24002

Rosa, M. R., Arana, R. S., \& Pereira, E. N. O impacto das tecnologias para o trabalho remoto na administração pública federal: um estudo com servidores técnicos administrativos em educação na UNILA. Práticas em Gestão Pública Universitária, 5(1), 196-217.

Rossi, M. A. G., \& Franzin, O. (2000). VPN-Virtual Private Network. GPr Sistemas/ASP Systemas.

Silva, V. M. B. D. (2012). Soluções Open-source para os Serviços de Fax e VPN numa Rede Empresarial.

Serapioni, M. (2000). Métodos qualitativos e quantitativos na pesquisa social em saúde: algumas estratégias para a integração. Ciência \& Saúde Coletiva, 5, 187-192.

Smith Heather A.; \& Mckeen, James D. (2006) IT in 2010: The Next Frontier. MIS Quarterly Executive. 5(3), 125-136.

Souza, C., \& Pimenta, D. (2014). Videoconferência e webconferência na EaD, análise dos usos e perspectivas de aplicação. In XI Congresso Brasileiro de Ensino Superior a distância. UNIREDE. 367-381.

Vieira, K. M., Postiglioni, G. F., Donaduzzi, G., dos Santos Porto, C., \& Klein, L. L. (2020). Vida de estudante durante a pandemia: isolamento social, ensino remoto e satisfação com a vida. EaD em Foco, 10(3). 